\title{
Improvement of Accuracy in Particle Extraction from Reconstructed Phase Image of Electron Holograms
}

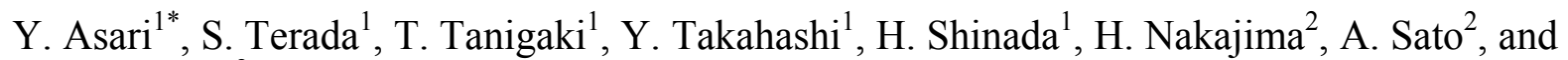 \\ Y. Murakami ${ }^{2}$ \\ 1. Research and Development Group, Hitachi, Ltd., Hitachi, Japan \\ 2. Department of Applied Quantum Physics and Nuclear Engineering, Kyushu University, Fukuoka, \\ Japan \\ * Corresponding author: Yusuke.asari.xy@hitachi.com
}

Electron holography [1] is known as a powerful tool to investigate electromagnetic fields formed by a target material. The study of the electromagnetic fields of the materials is one of the important subjects in the field of microscopy, because that provides information considered to be directly related to the origin of physical or chemical properties such as catalytic activities of nanoparticle catalysts. However, in the electron holography, the signal-to-noise ratio is not sufficiently high to obtain the essential information about the catalytic property of the nanoparticles.

One of the method for improving the $\mathrm{S} / \mathrm{N}$ ratio of nanoparticles can be an image processing like accumulation of a lot of images. That was formulated in the single-particle-analysis [2] for organic particles such as ionic channels or viruses. This type of $\mathrm{S} / \mathrm{N}$ improvement had not been performed for the electron holography. In the previous work [3], we prepared spindle-type $\mathrm{Fe}_{2} \mathrm{O}_{3}$ nanoparticles as a model system, and manually extracted the 493 particle images from 63 reconstructed phase images of electron hologram obtained by HF-3300X (Hitachi High-Technologies Co.). The extracted images were classified into 10 groups by using a convolutional neural network. By averaging a part of the images, we had succeeded to show that the background noise can be reduced even for the inorganic nanoparticles.

To obtain a sufficient accuracy in that kind of noise reduction method, huge numbers of image has to be processed automatically. But there are several difficulties, especially in the particle extraction. Here, two difficulties are focused. First one is a failure in picking up of particles caused by the weak contrast related to the weak electromagnetic fields of the materials. The other one is a detection error of particles (i.e. false positive; FP) occurs beside biprism area as shown Fig. 1 (a). Latter kind of failure is unique for the electron hologram. To overcome these difficulties, in this work, we developed the method for automatic particle extraction from the reconstructed phase image of electron hologram.

We explain our procedure of the particle extraction from the reconstructed phase image. In our method, first the reconstructed phase image is binarized. Next we calculate the area of each black island, and pick up the island having a certain area as a particle. In the binarization of the image a threshold has to be determined. The conventional global thresholding always fails like Fig. 1 (b). Thus we calculated local threshold according to the adaptive thresholding method [4]. The local threshold $T(x, y)$ is calculated by

$$
T(x, y)=m(x, y)[1+k s(x, y)-k],
$$

where $m$ is the mean value in the local region around $(x, y), s$ is the standard deviation, and $k$ is a constant. By using this local threshold each particle is clearly binarized like Fig. 1(c) and we can automatically obtained 213 images of particles from 63 reconstructed phase images.

In the extracted 213 particles we found that 30 images are picked up from both sides in the reconstructed phase image. In the both sides real particles are absent, because those are just unphysical patterns produced by inverse Fourier transformation. Thus that is the misrecognition of the particle, like the region pointed by dotted square in Fig. 1 (c). We call that kind of image "artifact" hereafter. 
Removal of the artifacts from the set of real particles is not so easy because we can not distinguish the artifacts and the real particles by using their area and contour as shown in Fig. 2 (a).

To remove the artifacts we originally defined the maximum gap between the neighboring pixel values $\Delta g_{\max }$ as

$$
\Delta g_{\max }=\arg \max _{(x i, y i)} \Delta g\left(x_{i}, y_{i}\right),
$$

where $\Delta g$ is a difference of the pixel value between neighboring pixel. By using the above equation, we can obtain a diagram of $\Delta g_{\max }$ for real particles and artifacts as shown in Fig. 2 (b). When the threshold is 0.05 , we found that the false positive can be avoided: i.e. The artifacts are completely removed.

In summary, we improved automatic particle extraction from the reconstructed phase image in electron holograms. By using combination of the adaptive thresholding and the newly defined maximum gap between the neighboring pixel values, we succeeded to remove artifacts from the extracted particle images. The improvement is also considered to be useful to reduce $\mathrm{S} / \mathrm{N}$ ratio.

\section{References:}

[1] D. Gabor, Nature 161, 777 (1948).

[2] J. Frank, Annual Review of Biophysics and Biomolecular Structure, 31 (2002) p.303.

[3] Y. Asari, et. al., International Microscopy Congress (2018) 372.

[4] J. Sauvola and M. Pietikainen, Pattern Recognition 33 (2000) p.225.

[5] The authors would like to thank Prof. A. Muramatsu and Prof. K. Kanie for providing samples and fruitful discussion. This work is partially supported by JST CREST Grant Number JPMJCR1664, Japan.
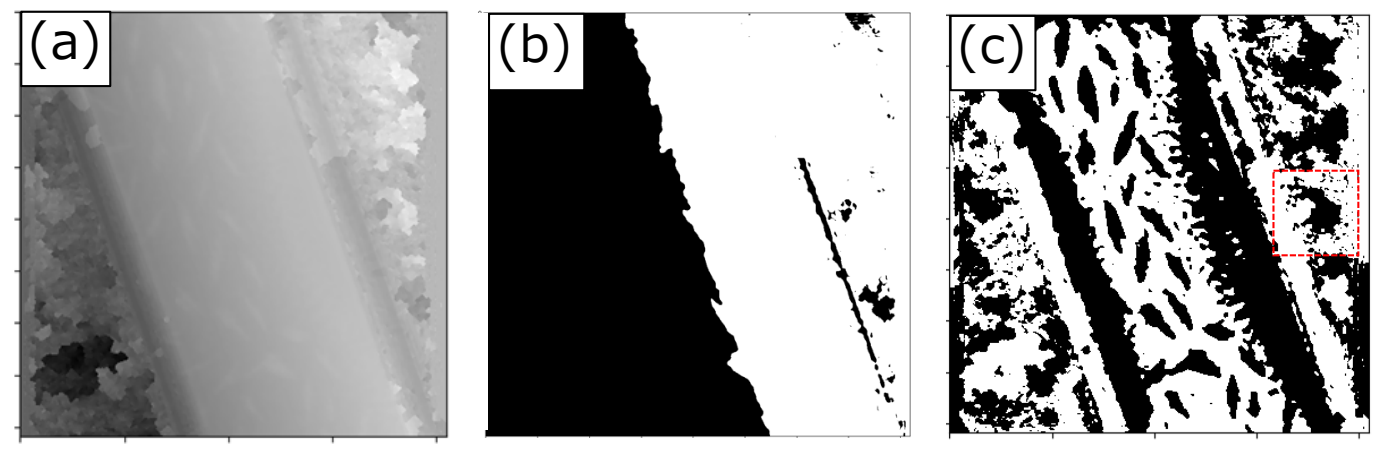

Figure 1. (a) Reconstructed phase image of electron hologram. Central zone is a biprism area. (b) Failure in binalization by using global threshold. (c) Binarized image of (a) by using adaptive threshold.
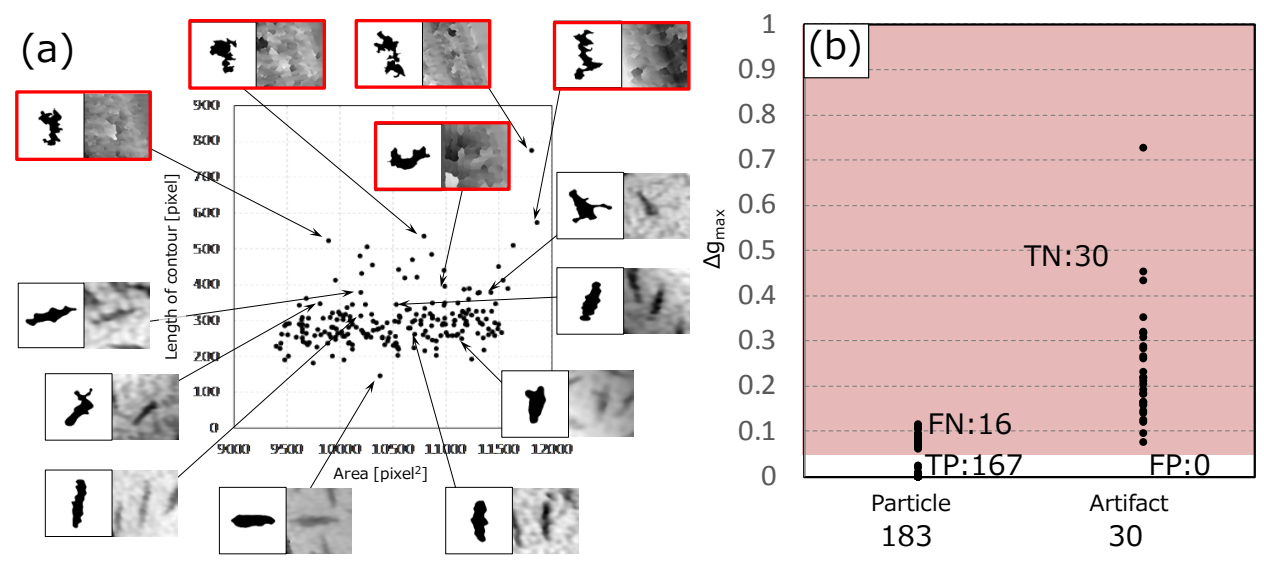

Figure 2. (a) Plot of extracted particles in an area-contour plane. The selected points are indicated by corresponding images (left: binarized, right: contrast enhancement). Artifacts are surrounded by red thick line. (b) Plot of $\Delta g_{\max }$. By using the threshold of $\Delta g_{\max }=0.05$, false negative will be zero. 